\title{
"Golden tube" with favorable vessel remodeling at four-year follow-up after bioresorbable vascular scaffold implantation for moderate to severe calcified lesions: Insights from intravascular imaging
}

\author{
Satoru Mitomo ${ }^{1}$, Luciano Candilio ${ }^{1,2}$, Akihito Tanaka ${ }^{1}$, \\ Azeem Latib $^{1}$, Antonio Colombo ${ }^{1}$ \\ ${ }^{1}$ Unit of Cardiovascular Interventions, IRCCS San Raffaele Scientific Institute, Milan, Italy \\ ${ }^{2}$ The Hammersmith Hospital, Imperial College London, United Kingdom
}

\section{Case 1}

A 66-year-old male underwent percutaneous coronary intervention (PCI) for diffusely calcified left anterior descending artery (LAD) (Fig. I). After predilatation, four bioresorbable vascular scaffolds (Absorb BVS, Abbott Vascular, Santa Clara CA) (distal: $2.5 / 18 \mathrm{~mm}$ and $2.5 / 28 \mathrm{~mm}$ with minimal overlap, middle: $3.0 / 28 \mathrm{~mm}$, proximal: $3.5 / 18 \mathrm{~mm}$ ) were implanted and postdilated with 1:1 diameter non-compliant (NC) balloons (24 atm) (Fig. II). Final intravascular ultrasound (IVUS) and optical coherence tomography (OCT) showed acceptable results. The narrowed diagonal ostium was left without additional intervention in view of no flow disturbance (Fig. a-c).

\section{Case 2}

An 87-year-old male underwent PCI for severely calcified stenosis in the mid LAD (Fig. i). After lesion preparation with rotational atherectomy and NC balloon, two BVSs (3.0/28 $\mathrm{mm}$ and $3.5 / 18 \mathrm{~mm}$ with minimal overlap) were implanted and postdilated with $3.5 \mathrm{~mm} \mathrm{NC}$ balloon (24 atm) (Fig. ii). Final IVUS demonstrated acceptable BVS expansion (Fig. $\mathrm{d}-\mathrm{f}$ ).

Four-year follow-up coronary angiography showed excellent results without significant restenosis (Fig. III, iii). OCT demonstrated complete BVS bioresorption and homogeneous neointimal coverage even on the segments with severe calcification and BVS overlapping (Fig. a'-f'). In the first case, the narrowing of the diagonal ostium had resolved and was free from scaffolds jailing with improvement of carina shift (Fig. c'). In the second case, IVUS showed lumen enlargement with partial plaque reduction when compared to the index procedure (Fig. d').

In severely calcified lesions, favorable vessel remodeling including homogeneous neointimal coverage of underling high-risk plaque, improvement of jailed side branch and lumen enlargement can be one of the potential benefits of BVS.

Informed consent was obtained from all individual participants included in the study. All procedures were in accordance with the ethical standards of the institutional research committee.

Conflict of interest: None declared

Address for correspondence: Antonio Colombo, MD, IRCCS San Raffaele Scientific Institute, Via Olgettina 60, 20132 Milan, Italy, tel: +390226437331, fax: +390226437339, e-mail: info@emocolumbus.it

Received: 06.11.2017 Accepted: 12.11.2017 


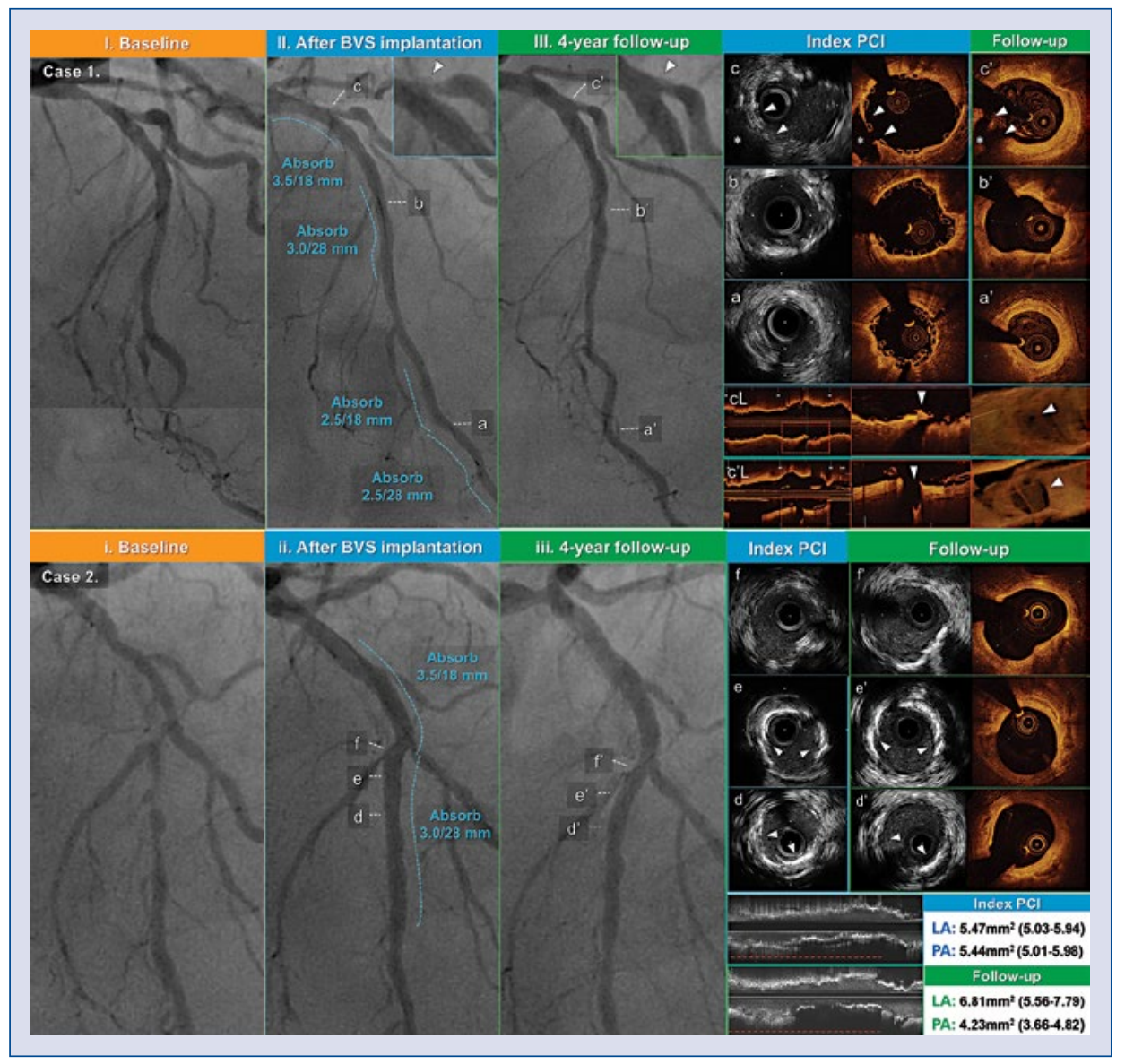

Figure 1. Coronary angiography (CAG) and intravascular imaging at the index procedure and 4-year follow-up after bioresorbable vascular scaffolds (BVS) implantation for severely calcified left anterior descending artery lesion (complete BVS bioresorption and homogeneous neointimal coverage). Case 1: upper panel; CAG: Baseline (I), after BVS implantation (II) and 4-year follow-up (III). Small panel in II and III: narrowed and improved diagonal ostium, respectively (arrow head). Intravascular images: Segments with BVSs overlap ( $a$ and $\left.a^{\prime}\right)$, severe calcification (b and b'), diagonal ostium ( $c$ and $c^{\prime}$ ), and optical coherence tomography longitudinal and 3-dimensional reconstruction images ( $\mathrm{cL}$ and $\mathrm{c}^{\prime} \mathrm{L}$ ). Arrow heads: narrowed diagonal ostium with carina shift and scaffold jailing (c and $\mathrm{cL}$ ) and improved diagonal ostium narrowing ( $c^{\prime}$ and $c^{\prime} \mathrm{L}$ ) (asterisk: diagonal). Case 2: lower panel; CAG: Baseline (i), after BVS implantation (ii), and 4-year follow-up (iii). Intravascular images: Segment with lumen enlargement with reduction of plaque area ( $d$ and $\left.d^{\prime}\right)$, severe calcification (e and $e^{\prime}$ ) and BVSs overlap ( $f$ and $\left.f^{\prime}\right)$. Arrow heads in $d$ and $d^{\prime}$ : segments with plaque area reduction. Lumen area (LA) and plaque area (PA) at the lesions indicated with red dashed lines in intravascular ultrasound longitudinal images (LA: $5.47 \mathrm{~mm}^{2}$ [5.03-5.94] at the index percutaneous coronary intervention (PCl), $6.81 \mathrm{~mm}^{2}$ [5.56-7.79] at 4-year follow-up, PA: $5.44 \mathrm{~mm}^{2}$ [5.01-5.98] at the index PCl, $4.23 \mathrm{~mm}^{2}$ [3.66-4.82] at 4-year follow-up, respectively). Arrow heads in e and $\mathrm{e}^{\prime}$ : calcium crackling. 